\title{
Théologiques
}

\section{Les rites : céder en résistant?}

\section{Guy Lapointe}

Volume 4, numéro 1, mars 1996

Les Rites : céder en résistant

URI : https://id.erudit.org/iderudit/602429ar

DOI : https://doi.org/10.7202/602429ar

Aller au sommaire du numéro

Éditeur(s)

Faculté de théologie de l'Université de Montréal

ISSN

1188-7109 (imprimé)

1492-1413 (numérique)

Découvrir la revue

Citer ce document

Lapointe, G. (1996). Les rites : céder en résistant? Théologiques, 4(1), 5-8.

https://doi.org/10.7202/602429ar d'utilisation que vous pouvez consulter en ligne.

https://apropos.erudit.org/fr/usagers/politique-dutilisation/ 
LIMINAIRE

\section{Les rites : céder en résistant?}

GuY LAPOINTE

Faculté de théologie

Université de Montréal

L'énigme des rituels est notre énigme et non pas celle des sociétés et des religions défuntes.

L'intitulé de ce numéro de Théologiques peut surprendre. Il requiert quelques éclaircissements. Le monde des rituels se présente, s'expose, s'impose tout à la fois à la vue et à l'ouie et semble garder pour lui, secrète, sa raison. Quelle est-elle? Conserver la mémoire des origines? Transmettre des valeurs sociales, culturelles ou religieuses? Ou plus globalement, produire des significations? Il semble pourtant que les rites imposent leur nécessité et résistent, moins pour produire des significations, que parce qu'il y a de la signification dans l'existence - et possiblement toujours trop de signification? Même si, à l'observation, certaines pratiques rituelles apparaissent parfois dépourvues de sens, se pourrait-il, qu'à même nos résistances à les pratiquer, on cède à leur nécessité parce qu'ils ouvrent et balisent un chemin de sens?

Le symbole "donne à penser» a pu écrire Paul Ricœur. Le rituel effectue ce «donner à penser ». Il reste une énigme tout aussi indéchiffrable (mystérieuse) que l'être humain l'est à lui même. À cet égard, le rite nous résiste par sa nécessité même, là où obligatoirement, les êtres humains cherchent et trouvent une pluralité de scènes pour jouer leur rôle. Mais comme le rite "opère à partir du sens qui est d'abord inconnu ou ignoré par la plupart » (G.-R. St-Arnaud, p. 90), il nous invite à leur céder pour parcourir ces chemins traversant le sens à venir, en même temps que, devant l'angoisse de l'inconnu et de l'étrangeté de la vie ellemême, ils rendent compte de nos résistances à les pratiquer. Vive tension 
inscrite dans la dynamique même du champ symbolique. Le rite : céder en résistant?

À un autre niveau, après le passage de Freud, et les interprétations reçues qu'on en a données, on a eu tendance à qualifier les rites tout autant comme des pratiques banales, que comme des impostures nourrissant l'aliénation de l'être humain. Depuis, les sciences humaines et la théologie tendent à redécouvrir qu'ils constituent une dimension fondamentale de l'être humain et de sa quête d'identité. Dans les dix dernières années, les chercheurs ont fait une re-lecture des travaux d'Emile Durkheim, produits au début de ce siècle. On s'est aperçu qu'il avait opéré en matière de ritologie un véritable renversement copernicien, ouvrant la voie à de nombreux ouvrages internationaux ${ }^{1}$. Les conduites rituelles n'étaient plus reléguées au chapitre des aberrations humaines ou, au contraire, exaltées comme on a pu le faire dans un certain catholicisme au nom du Mystère et de l'Obscur. Les conduites rituelles étaient, d'une part, analysées, dégagées d'une gangue de présupposés positifs et négatifs, et, d'autre part, placées dans un cadre conceptuel. Au-delà de toute autre considération, on s'est aperçu, à même les bouleversements de la modernité, que les rituels sociaux et religieux ont la vie dure, même si le discours théorique, lui, se cherche parfois désespérément. En ce sens, les rituels résistent et persistent sous de multiples et déroutantes recompositions de leurs mises en scène à même l'évolution des sociétés et des systèmes religieux. Les chercheurs ont compris que les groupes humains, comme par un instinct de survie, finissent toujours par céder devant leur nécessité. Après un temps de résistance à vouloir saisir cette énigme, ils mettent de plus en plus d'énergie à vouloir les approcher et à construire un cadre théorique pour rendre compte de cette réalité jamais circonscrite dans un discours. On n'a qu'à penser aux mutiples travaux publiés et aux colloques qui se tiennent depuis quelques années sur cetre question. Les éléments bibliographiques qui accompagnent les contributions à ce numéro l'illustrent hautement.

D'autres considérations ont aussi prévalu dans l'élaboration de cette thématique. Si toute religion s'exprime nécessairement dans des rituels, il ne s'ensuit pas que la ritualité soit tout entière circonscrite par la croyance et que tout rite soit nécessairement le résidu de quelque sécrétion religieuse. Pourtant, on ne se trompe vraisemblablement pas en pensant que l'humanité tout entière se serait d'abord exprimée religieusement par des gestes rituels, la parole apparaissant alors comme l'accompagne-

1 Les formes élémentaires de la vie religieuse. Paris, Alcan, 1912. 
ment, le commentaire qui se greffe sur les pratiques religieuses et sociales $^{2}$. À cet égard, les rituels sont sans doute la forme la plus archaïque de l'expression humaine. La religion reste un aspect particulier mais nécessaire de l'aventure symbolique des sociétés humaines. Ce numéro a voulu maintenir sous un même regard la religion et l'activité symbolique des sociétés.

Il est tout à fait normal qu'une revue liée à une Faculté de théologie chrétienne se préoccupe d'entrer dans le champ d'une réflexion pluridisciplinaire sur les rites. Les rituels ne furent-ils pas et ne sont-ils pas encore des lieux ambigüs, certes, mais significatifs, de la transmission des traditions sociales, culturelles et religieuses? En plus de la mémoire inscrite dans l'art, dans le discours théologique et catéchétique et dans des réalisations caritatives d'envergure, comme autant de vestiges d'un passé vigoureux, le réseau rituel, parfois tant suspecté par les responsables des Églises, tient le coup et empêche les générations de sombrer dans l'oubli, du moins lors des grands événements de la vie familiale et de la collectivité qui se réclament de la tradition chrétienne. On pourrait très vraisemblablement en dire tout autant pour les autres grandes traditions religieuses dans le monde. À même les évolutions parfois déroutantes des réseaux religieux et de ceux des sociétés, les rituels, comme en une sorte d'entêtement, ont la vie dure, et même se renouvellent. C'est une énigme qui rejoint l'énigme même de l'être humain. On avait l'habitude en théologie catholique de produire de longs développements, parfois apologétiques, sur l'efficacité des sacrements chrétiens, sur leur performativité, comme on dit maintenant. Cette sorte d'intuition, liée à l'opérationnalité de la grâce de Dieu, trouve, depuis quelques années, à la lumière d'une recherche interdisciplinaire de plus en plus féconde sur les rites, une confirmation. Personne aujourd'hui ne contestera l'efficacité des rituels quels qu'ils soient.

L'énoncé de notre thème, "les rites: céder en résistant", indique également la tension dans le processus même du rituel puisqu'il s'agit de mises en scène du passé dans un présent qui cherche un avenir. C'est une sorte de mise en scène à la fois de l'avenir d'un souvenir (In illo tempore) et d'un souvenir de l'avenir. Telle est la raison pour laquelle, même en observant les déplacements du religieux dans la modernité, les rituels se recomposent à même des réalités parfois inédites. Ils sont la marque d'une "origine imprenable", comme aimait à le dire Michel de Certeau. C'est

2 Henri HATZFELD, Les racines de la religion. Tradition, rituel, valeurs. Paris, Seuil, 1993, p. 104. 
pourquoi le rite est appel au sens, il renvoie toujours à un autre, à de l'autre. D'ailleurs, l'ensemble des contributions à ce numéro poursuivent des réflexions extrêmement significatives pour enrichir les cadres critiques autour des rites.

Le sociologue Raymond Lemieux ouvre le débat en montrant comment en plein cœur de la sécularité, les rites, surtout ceux liés aux grandes traditions chrétiennes, sont repris, réinterprétés, bricolés et reçoivent des significations inédites et souvent imprévisibles. Que reste-til des rites après Jésus? Une réflexion de l'exégète Jean-Paul Michaud, lequel, à partir d'une re-lecture fascinante de l'épître aux Hébreux, questionne profondément la position et le sens des pratiques rituelles dans le christianisme, et surtour leur relation à l'éthique. Eric de Rosny illustre, en décrivant, pour ensuite la commenter, une expérience d'une mise en scène rituelle vécue au Cameroun, la résistance et la résurgence des rites traditionnels dans l'Afrique moderne. Il montre également la fragilité des rituels chrétiens et leur recomposition à même les rites africains traditionnels. Freud a semblé porter un coup mortel à la pertinence de la pratique des rituels religieux. Guy-Robert St-Arnaud reprend, avec une grande finesse, la problématique freudienne, pour aider à mieux saisir le sens des critiques que ce psychanalyste adresse aux pratiques rituelles religieuses. Enfin, Denis Jeffrey, en jetant un nouveau regard sur les ritualités religieuses, s'attache, entre autres, à montrer la fonction'transgressive des rituels qui mettent en scène des interdits.

On sera peut-être surpris, à la lecture de l'ensemble de ce numéro de Théologiques, de ne pas trouver au moins une contribution explicite sur les sacrements chrétiens. D'une part, un article ne nous est pas parvenu; d'autre part, tous les auteurs touchent explicitement la question des sacrements chrétiens, en particulier ceux de Raymond Lemieux et de Jean-Paul Michaud. Comme dans tous les rites - et c'est aussi leur singularité - les sacrements chrétiens parlent, pour le meilleur et pour le pire, de l'origine. Ils font mémoire du Corps absent en même temps que du corps d'Église. Ils mettent en scène des images de Dieu qu'ils contribuent à construire dans l'imaginaire croyant. En terminant, il est facile d'observer que, dans la pratique, s'il y a souvent de la résistance à certaines pratiques sacramentelles, on cède devant les rituels liés à des événements significatifs de la vie. Il faudra certainement revenir sur ce point. Pour l'heure, nous nous trouvons devant une pluralité d'approches, d'éclairages convergents sur un même objet. Sont-ils complémentaires? Aux lectrices et aux lecteurs d'en juger. 